\title{
Compact Fixed-exit UHV DCM for XAFS
}

\author{
K. Rickers, U. Brüggmann, W. Drube, M. Herrmann, J. Heuer, E. Welter, \\ H. Schulte-Schrepping and H. Schulz-Ritter
}

\author{
Hamburger Synchrotronstrahlungslabor HASYLAB am Deutschen Elektronen-Synchrotron DESY \\ Notkestr. 85, D-22607 Hamburg, Germany
}

\begin{abstract}
A double-crystal, UHV-compatible monochromator for XAFS applications at bending magnet beamlines has been designed. It uses two crystal sets, $\mathrm{Si}(111)$ and (311), on a common central rotation axis driven by an ex-vacuo goniometer. All mechanical and electrical components are mounted on a $400 \mathrm{~mm}$ UHV flange which is attached to a compact vacuum chamber. The first crystals are water cooled using connector- and bellowless tubing through the fluidic sealed feedthrough of the central rotation. The first crystal set is mounted off-axis and can be translated vertically to keep the fixed exit condition. The second crystal set uses small crystals of the same size as the first. In order to accept the reflected beam of the first crystal at small Bragg angles, it is tangentially translated along the beam. The angle can be varied from $5^{\circ}$ to $55.5^{\circ}$ resulting in a total energy range $2.4-43.4 \mathrm{keV}$ for $\mathrm{Si}(111) /(311)$. Crystal sets are interchangeable by translating the vacuum chamber. Angle encoding is achieved by a Renishaw incremental optical encoder in vacuo.
\end{abstract}

Keywords: Double crystal monochromator, XAFS

PACS: $41.50 .+\mathrm{h} ; 41.60 . \mathrm{Ap} ; 07.85 . \mathrm{Qe} ; 61.10 \mathrm{Ht}$

\section{INTRODUCTION}

At the DORIS III storage ring, several bending magnet beamlines are dedicated to X-ray absorption spectroscopy. Depending on the beamline optics and the main fields of application, the energy range is either preferentially "soft", i.e. starting from the Sulphur K-edge at $2.4 \mathrm{keV}$ going up to approximately $11 \mathrm{keV}$, or "hard", i.e. from around $5 \mathrm{keV}$ up to beyond $40 \mathrm{keV}$. High photon energies are available at DORIS III bending magnets with considerable flux due to their high critical energy $\left(E_{c}=16.6 \mathrm{keV}\right)$. The beamlines and monochromators have been implemented many years apart and their layout is quite different. Based on the long standing experience with a variety of different double crystal monochromators at the laboratory, the goal was to design a new device which incorporates as many of the advantages and features of the other instruments as possible. At the same time, it should be compact and UHV-compatible to allow its straightforward integration into existing bending magnet beamlines without their major modification.

\section{DESIGN AND PERFORMANCE}

The device should be used primarily, but not exclusively, for XAFS experiments at bending magnet beamlines. Therefore, the main design criteria were a wide energy range, fast tunability as well as fixed-exit operation. To cover the energy range from just below the Sulphur K-edge and to above $40 \mathrm{keV}$, it seemed advisable to implement two different Si crystal pairs, (111) and (311), interchangeable in-situ by remote control. Fast and precise tunability is best reached using a common rotation stage for both crystals which has proven to be suitable for fast continuous scanning absorption measurements (QEXAFS)[1]. A corresponding (non fixed-exit) monochromator has been in operation at HASYLAB for many years [2] and is very successfully used for in-situ catalysis XAFS studies.

For the new device, an optical design proposed by Mills, King and Lee [3,4] was adapted which allows fixed-exit operation. This is accomplished by an off-rotation-axis mount of the first crystal, which is translated perpendicular to the beam. The original design uses a fixed second crystal which is long enough to reflect the beam at all accessible Bragg angles. Here it was decided to use a shorter crystal and to implement its tangential translation along the beam instead. A short crystal makes it easier to ensure perfect crystal properties across the entire surface. The sizes used here are $40 \mathrm{~mm} \times 40 \mathrm{~mm}$ for both crystal pairs. It is noted that during a typical XAFS energy scan covering 1-2 keV, the second crystal is not moved to minimize mechanical instabilities. Prior to an energy scan the crystal is positionend appropriately by the software to maximize the available scan range (for decreasing Bragg angles during the scan). The Bragg angle range was limited to $5^{\circ}-55.5^{\circ}$ which corresponds to an energy range from $2.4-22.6 \mathrm{keV}$ for $\mathrm{Si}(111)$

CP879, Synchrotron Radiation Instrumentation: Ninth International Conference, edited by Jae-Young Choi and Seungyu Rah

(C) 2007 American Institute of Physics 978-0-7354-0373-4/07/\$23.00 


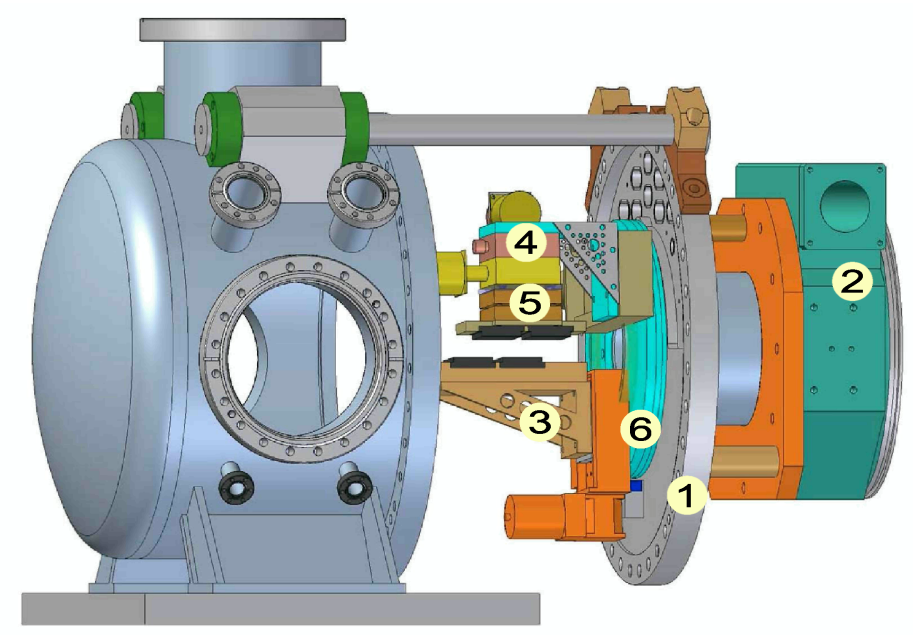

FIGURE 1. Schematic of the monochromator, shown in its "service" position. All components are attached to an O-ring sealed $400 \mathrm{~mm}$ UHV-flange (1). The outside goniometer (2) is coupled by a hollow-shaft ferrofluidic $\mathbb{R}$ sealed feedthrough to the common rotation in-vacuo. The first crystal pair (3) can be moved perpendicular to the beam and is indirectly water-cooled. The second crystal pair is mounted on a two-axis cradle (4) for alignment relative to the first, the unit can be translated along the beam. A piezo-driven micro-goniometer (5) allows to fine tune the relative Bragg angles. The angle position is read out by a Renishaw ${ }^{\circledR}$ optical encoder system (6).

and 4.6-43.4 keV for $\mathrm{Si}(311)$. To keep the dimensions of the vacuum chamber small, the central rotation is driven by a goniometer (Huber 420) from the outside, coupled by a hollow-shaft ferrofluidic ${ }^{\circledR}$ vacuum feedthrough [5] with $70 \mathrm{~mm}$ inner diameter (see Fig. 1). Precise angular encoding is achieved by an in-vacuum Renishaw [6] incremental optical encoder system. All mechanical components are mounted on the central rotation in vacuo, electrical connectors and feedthroughs are provided on the common $400 \mathrm{~mm}$ UHV flange. The entire unit can be easily retracted for maintenance.

The first crystal pair is indirectly water-cooled. The thermal coupling between the crystals and the copper heat sink is achieved by Ga-In eutecticum (Gallistan $\left.{ }^{\circledR}\right)$. Side-cooling was not considered necessary because thermal gradients are small (total power on the crystal $\approx 100 \mathrm{~W}$ ) and the simpler design was preferred. The cooling water supply was designed without water-to-vacuum joints such as brazing and connectors (Fig. 2). In order to enable crystal height tuning without the use of bellows, the flexibility of the (comparatively long) copper tubings is utilized which pass through the hollow shaft of the rotational axis.

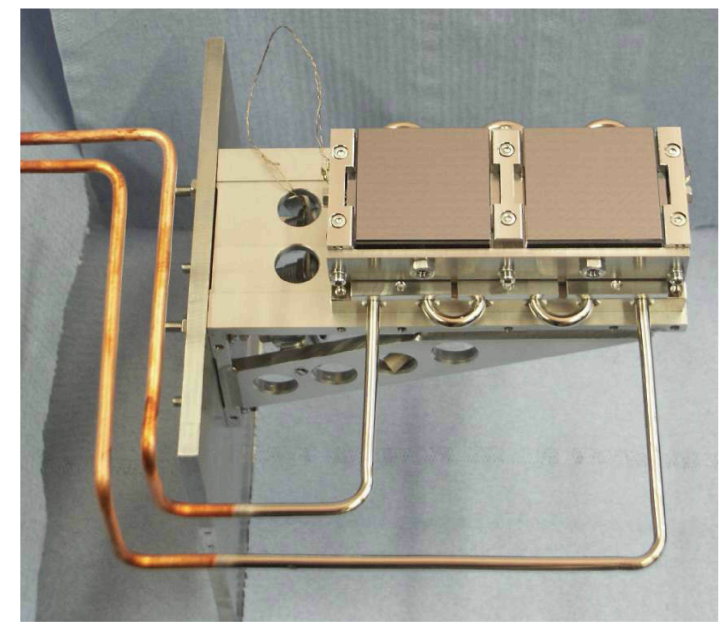

FIGURE 2. First crystal stage with attached indirect water cooling (Si(111) and (311), crystal size $40 \times 40 \mathrm{~mm}^{2}$ ). The tubing passes through the hollow shaft rotational axis to the left. 


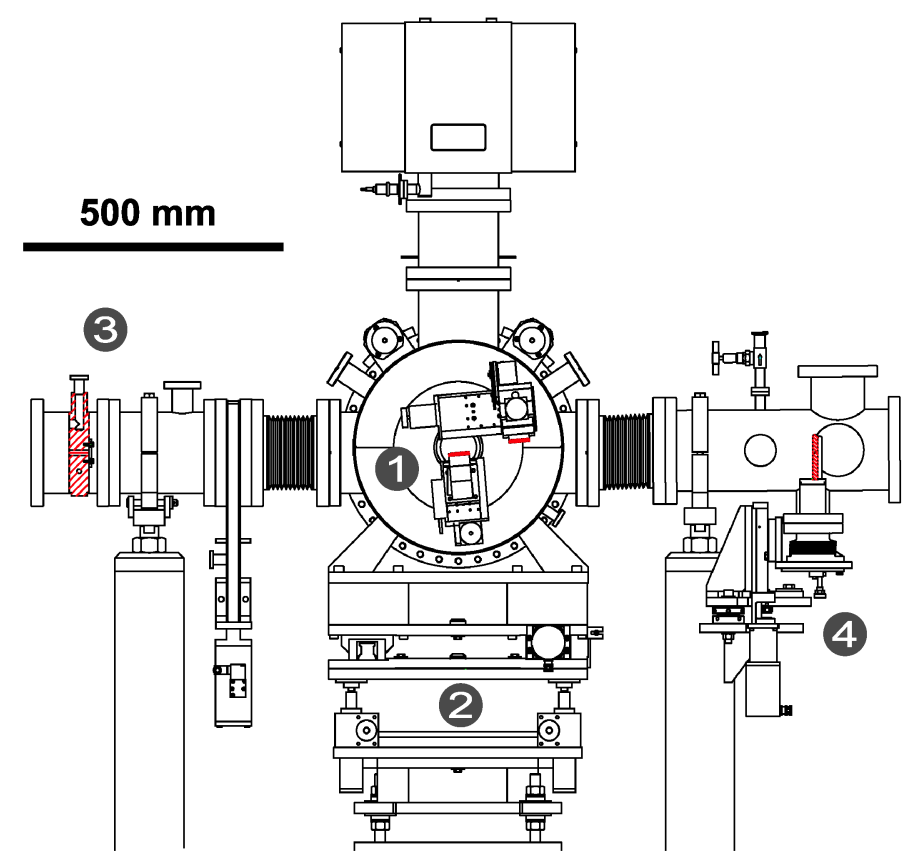

FIGURE 3. Schematic showing the monochromator (1) integrated into beamline C at DORIS III. The outer flange-to-flange distance of the vacuum chamber is $500 \mathrm{~mm}$ allowing it to be mounted onto a standard HASYLAB mirror chamber support (2) which provides additional degrees of freedom such as translation perpendicular to the beam (for crystal change) and height adjustment. The incoming white beam is passing through a fixed width carbon window (3) to separate the vacua and to cut off the low energy spectrum. Behind the monochromator, a water-cooled adjustable absorber (4) with a pinhole opening is used to either block the direct beam if the crystals are used for monochromatization or to pass the white beam through the pinhole on to the experiment if the crystals are moved out of the beampath.

Because of its compactness the monochromator vacuum chamber easily fits onto a standard (HASYLAB) mirror chamber support which offers translational (and optionally also rotational) degrees of freedom (Fig. 3). Therefore, it is straightforward to bring the different crystals into the beam by translating the vacuum chamber $(50 \mathrm{~mm})$ which is connected to the beamline by flexible bellows. Inlet and outlet flanges are large enough (150 $\mathrm{mm}$ conflat) to pass the beam through in both positions. The chamber height may be adjusted as well although this is only necessary once during the initial alignment. A complete crystal change, including relative $\Theta$-angle re-adjustment takes about 2 minutes and is remotely done by the controlling software. An adjustable absorber with a pinhole behind the monochromator allows to pass the white beam on to the experiment. Therefore, both crystals can be easily moved out of the direct beam. A Ta wire, attached to the absorber, can be scanned through the monochromatic beam to measure its position.

In addition to pumping through the beamline, a $300 \mathrm{l} / \mathrm{s}$ ion pump on the top of the monochromator vacuum vessel is used to maintain a base pressure of $3 \cdot 10^{-8}$ mbar without bake-out in "stand-by" operation, i.e. when no motors are moving. During motor movements the pressure will increase slightly because of additional degassing due to components warming up.

The initial performance tests of the instrument were very promising. While scanning, the relative detuning of the crystals is controlled by a digital monochromator feedback system (MOSTAB). The piezo driven micro-goniometer (maximum detuning $600 \mu \mathrm{rad}$ ) was mounted onto the second crystal stage, instead of the first one, to enhance the relative energy stability in the monochromatic beam under detuning conditions $[7,8]$.

The monochromator has been designed for fixed-exit operation which is - of course - achieved for conventional step-by-step scanning; the offset between white and monochromatic beam is $20 \mathrm{~mm}$. For fast scanning measurements (QEXAFS) the motors are continuously moving and their positions are recorded "on the fly". Unfortunately, the present motor controllers cannot be programmed with non-linear step profiles. Therefore, all motors move linearly which causes a certain deviation from the fixed-exit condition. This is shown for two typical examples, Ti and $\mathrm{Cu} \mathrm{K}$-edges, in Fig. 4. It is noted that for small Bragg angles the effect becomes practically negligible. However, at large Bragg angles, e.g. at the $\mathrm{S} \mathrm{K}$-edge, the deviation is significant (may exceed $1 \mathrm{~mm}$ ) so that continuous scanning may be problematic. 


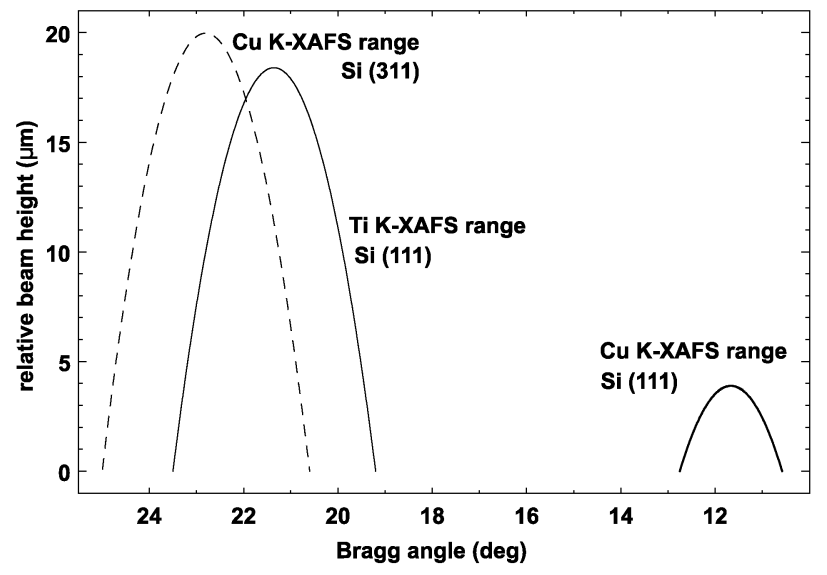

FIGURE 4. Deviation of the exit beam height during continuous scanning in the range of two typical K-absorption edges, Ti K-edge scanned with $\mathrm{Si}(111)$ (range: 4958 to $6011 \mathrm{eV}$ ) and $\mathrm{Cu}$ K-edge scanned with $\mathrm{Si}(111)$ and $\mathrm{Si}(311)$ (range: 8958 to 10760 $\mathrm{eV})$. At the larger Bragg angles the maximum relative offset is below $20 \mu \mathrm{m}$ and it becomes practically negligible $(<4 \mu \mathrm{m})$ in the case of the $\mathrm{Cu}$ K-edge measured with $\mathrm{Si}(111)$.

To demonstrate the performance of the monochromator, a $\mathrm{Cu}$ metal $\mathrm{K}$-edge spectrum obtained in the continuous scanning mode is shown in Fig. 5. The initial tests have shown that it is indeed performing very well as expected and it is planned to gradually replace some of the older systems at DORIS III bending magnet beamlines with this type of instrument.

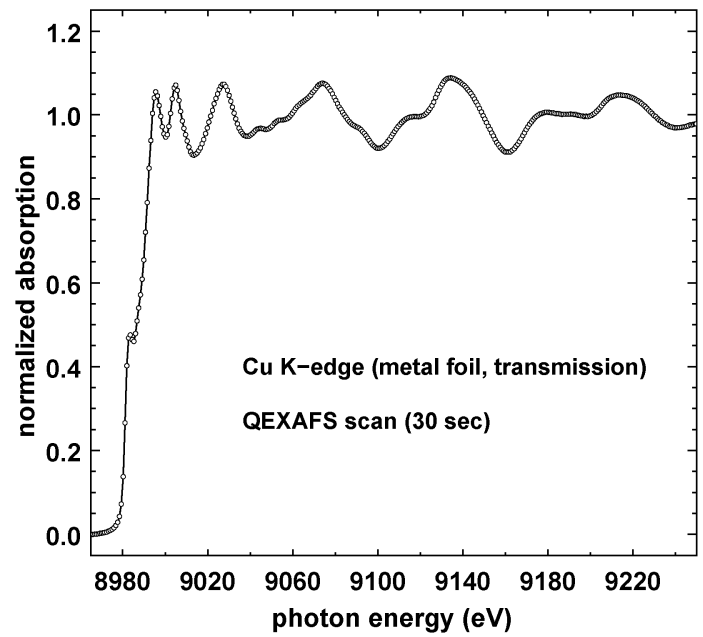

FIGURE 5. Cu K-edge absorption spectrum (background subtracted and normalized) measured from a metal foil in transmission $(\mu \mathrm{d}=2.3)$ using $\mathrm{Si}(111)$ in the continuous scanning mode. The sampling time per data point is $35 \mathrm{~ms}$, the total scan time $\approx 30 \mathrm{~s}$.

\section{REFERENCES}

1. R. Frahm, Rev. Sci. Intrum. 60, 2515 (1989)

2. R. Frahm, HASYLAB Annual Report 1995, p. I-113 (1996).

3. D.M. Mills and M.T. King, Nucl. Instr. Methods 208, 341 (1983).

4. W.K. Lee and D.M. Mills, ANL/APS/TB-4 (February 1993).

5. Ferrotec Corporation (http://www.ferrotec.com).

6. Renishaw plc, New Mills, Gloucestershire, UK (http://www.renishaw.com)

7. H. Schulte-Schrepping and W. Drube, Nucl. Instr. Methods A 467, 396 (2001).

8. Z.C. Hou, Rev. Sci. Intrum. 76, 013305 (2005). 